\title{
Mamma-Ca.-Staging: besser mit PET/CT
}

\begin{abstract}
Die Prognose des lokal fortgeschrittenen Mammakarzinoms bleibt infaust. Die Frage ist, ob sie durch initiales Staging mit der 18Fluor-(F)Fluordesoxyglukose(FDG)-Positronen-Computer-Tomografie (PET/CT) verbessert werden kann.
\end{abstract}

- Nuklearmediziner der kommunalen Krankenhäuser in Paris untersuchten prospektiv über fünf Jahre 117 Patienten mit fortgeschrittenem, entzündlichem $(\mathrm{n}=35)$ und nicht entzündlichem $(\mathrm{n}=82)$ Mammakarzinom. Sie wurden mit konventionellen Methoden (Knochenscan, Mammografie, Sonografie des Abdomens und Beckens sowie Standardtomografien) diagnostiziert. Abschließend erhielten sie eine 18F-FDG-PET/CT, deren Resultate von externen Spezialisten eingeschätzt wurde. Die Resultate des 18F-FDG-PET/ CT wurden mit denen der CT-Standardmethoden verglichen.

Mit der 18F-FDG-PET/CT wurden alle Primärtumoren identifiziert. Die Scans bestätigten den Lymphknotenbefall der IIIC-Patienten und markierten einen unerwarteten Lymphknoten-Befall (infra- und supraklavikulär, mammaintern) bei 32 zusätzlichen Patientinnen. Ferne Metastasen (Knochen, Leber, Lunge, Pleuren) wurden bei 43 Frauen sichtbar. Die konventionellen Methoden hatten sie nur bei 28 Patientinnen erkannt. Die 18F-FDG-PET/CT veränderte insgesamt das Tumorsta- dium bei 61 (52\%) von 117 Patienten und damit auch die Therapie.

\section{- D. Groheux et al. \\ 18-F-FDG PET/CT in staging patients with locally advanced or inflammatory breast cancer: comparison to conventional staging. J Nucl Med 2013;54: 5-11; doi: 10.2967/jnu. med.112106864}

\section{Kommentar}

Die 18F-FDG-PET/CT hat den Vorteil, dass sie die Kontrolle der Metastasierung von Thorax, Abdomen und Knochen in einer Sitzung erlaubt. Alle distalen und weitere zusätzlichen Läsionen wurden nachgewiesen. Diese Methode ist ein wichtiger Schritt in Richtung einer individuellen Therapie, so die Autoren.

K. MALBERG =

\section{Polypen entfernen senkt Mortalität}

Die endoskopische Entfernung adenomatöser Kolonpolypen verringert das Mortalitätsrisiko des kolorektalen Karzinoms.

- Zwischen 1980 und 1990 unterzogen sich über 9100 Patienten im mittleren Alter von 62 Jahren einer diagnostischen Kolonoskopie. Bei 3778 wurde eine endoskopische Polypektomie von Adenomen und Nichtadenomen durchgeführt. Anhand des nationalen Sterberegisters

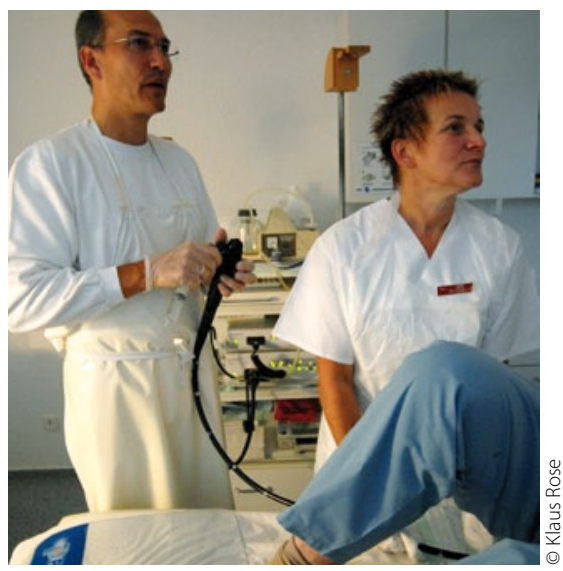

Kolonoskopische Polypektomie senkt die Sterblichkeit am Kolonkarzinom. versuchte man, im Zeitraum bis zu 23 Jahren nach dem Eingriff festzustellen, welche Personen verstorben waren und was die Todesursache war. Die Mortalität am kolorektalen Karzinom wurde dabei mit der erwarteten inzidenzbasierten Mortalität in der Allgemeinbevölkerung verglichen. Von $2602 \mathrm{~Pa}$ tienten mit Adenomen, die im Rahmen der Studie entfernt worden waren, waren 1246 innerhalb einer medianen Beobachtungsdauer von 15,8 Jahren verstor- ben. Bei zwölf war die Todesursache ein kolorektales Karzinom. Nach der allgemeinen Mortalitätsstatistik in der Bevölkerung wären 25,4 Todesfälle am kolorektalen Karzinom zu erwarten gewesen. Mit der kolonoskopischen Polypektomie gelingt also eine 53\%ige Reduktion der Mortalität.

\section{- A. G. Zauber et al.}

Colonoscopic polypectomy and long-term prevention of colorectal-cancer deaths. New Engl. J. Med. 2012;366: 687-696

Kommentar

Auch wenn die National Polyp Study keine Aussage zur Effizienz der ScreeningKoloskopie in der Allgemeinbevölkerung machen kann, so belegt sie doch den positiven Effekt einer Entfernung von Adenomen auf die Mortalität des kolorektalen Karzinoms. Streng genommen müsste der wissenschaftliche Beweis für die Effektivität der Polypektomie zwei Kohorten von Patienten mit Adenomen umfassen, die bei der einen Kohorte entfernt, bei der anderen nur beobachtet werden. Eine derartige Vergleichsstudie wäre allerdings aufgrund des bekannten malignen Potenzials von Adeno- men nicht vertretbar. Kritiker der ScreeningKolonoskopie wenden allerdings ein, dass bei diesem Verfahren auch zahlreiche nichtadenomatöse Polypen entfernt werden, die sich nie zu einem Karzinom entwickelt hätten. Die gesundheitspolitisch aufwändige Screening-Koloskopie wird sich langfristig nur halten lassen, wenn tatsächlich eine Senkung der Mortalität des kolorektalen Karzinoms gezeigt wird. Bis die Ergebnisse einer randomisierten und kontrollierten Studie der Screening-Kolonoskopie vorliegen, müssen wir uns an die hier veröffentlichten Daten halten.

H. S. FÜESSL = 